\title{
Transcranial Yellow, Red, and Infrared Laser and LED Stimulation: Changes of Vascular Parameters in a Chick Embryo Model
}

\author{
Gerhard Litscher $^{a-c, f, g} \quad$ Lequan Min $^{f}$ Christina Angelika Passegger ${ }^{d}$ \\ Daniela Litscher ${ }^{a-c} \quad$ Min Li $^{f} \quad$ Mian Wang $^{f}$ Nassim Ghaffari-Tabrizi-Wizsy $^{d}$ \\ Ingeborg Stelzer $^{d}$ Georg Feigl ${ }^{\mathrm{e}}$ Ingrid Gaischek ${ }^{\mathrm{a}-\mathrm{c}}$ Guangjun Wang ${ }^{\mathrm{g}}$ \\ Anton Sadjak $^{d}$ Frank Bahr $^{\text {h }}$ \\ ${ }^{a}$ Research Unit for Complementary and Integrative Laser Medicine, ${ }^{b}$ Research Unit of \\ Biomedical Engineering in Anesthesia and Intensive Care Medicine, ${ }^{\mathrm{C}} \mathrm{TCM}$ Research \\ Center Graz, ${ }^{d}$ SFL Chicken CAM Lab, Institute of Pathophysiology and Immunology, and \\ e Institute of Anatomy, Medical University of Graz, Graz, Austria; ${ }^{f}$ Department of Information \\ and Computing Science, School of Mathematics and Physics, University of Science and \\ Technology, and Institute of Acupuncture and Moxibustion, China Academy of Chinese \\ Medical Sciences, Beijing, China; ${ }^{\mathrm{h}}$ German Academy of Acupuncture and European \\ Academy of TCM, Munich, Germany
}

\section{Key Words}

Transcranial laser stimulation · Chick embryo model · Chorioallantoic membrane · Blood vessels · Image matting algorithm · Yellow laser · Red laser · Infrared laser · Light-emitting diode

\section{Abstract}

Background: The use of transcranial laser or light-emitting diode (LED) therapy in the treatment of different neurological diseases is attracting increasing attention. The main goal of this study was to investigate different kinds of scientifically and commercially available laser (yellow, red, and infrared) and LED stimulation systems for the first time using a chick embryo model under a fixed human cadaver (scalp and/or skull) and without this anatomical preparation. Methods: In the present study, the extra-embryonic vasculature of a 1-week-old chick embryo chorioallantoic membrane (CAM) was investigated. For stimulation, four different systems were used (infrared LED and yellow, red, and infrared laser). Four measurement periods were compared: before stimulation, immediately after stimulation onset, immediately after the 10-min stimulation was turned off, and 6 min after stimulation had ended. Special image processing programs developed at the Beijing University of Science and Technology were used for data analysis. Results: The different kinds of laser induced different effects, e.g. the direct stimulation with yellow laser led to initial vasoconstriction $(-17 \%)$, whereas yellow 
laser stimulation through a human skull resulted in an increase in blood volume. These effects could only be observed after mathematical processing of the images. Conclusion: After intensive basic research (e.g. CAM model), transcranial laser stimulation may open up new therapies for lifestyle-related diseases such as stroke, dementia, Alzheimer's or possibly Parkinson's disease.

(C) 2015 S. Karger AG, Base

\section{Introduction}

Integrative medicine has become an indispensable part of our health care management system, and it is known to cover a wide range of therapies which vary from culture to culture [1]. Basic research on integrative medicine has been successfully performed in many centers worldwide, using a broad spectrum of innovative methods [2].

Neurological diseases will become a very important topic in research because of the movement in the age pyramid. For example, it was stated in review articles that by 2030 an estimated 75.6 million, and by 2050 even 135.5 million, people will suffer from dementia alone [3]. Therefore, intensive basic and clinical research on this topic is necessary [4].

The use of transcranial laser or light-emitting diode (LED) therapy in the treatment of different neurological diseases is attracting increasing attention. The stimulation can have neuroprotective and positive cognitive effects in animals as well as in humans [5]. Recently, it has also been shown in an animal study that transcranial low-level laser therapy enhances learning, memory, and neuroprogenitor cells after traumatic brain injury in mice [6]. In contrast, there are also publications which do not recommend this kind of treatment in different neurological diseases. In their 2014 study, Hacke et al. [7] stated that transcranial laser therapy does not have a measurable neuroprotective effect in patients with acute ischemic stroke when applied within $24 \mathrm{~h}$ after stroke onset. Because of such controversial opinions, basic research is absolutely necessary to achieve more insight and understanding of light propagation through tissues including the scalp, skull, meninges, and last but not least the brain. Only very few scientific investigations are available in this area of research $[3$, 8-14].

The main goal of this study was to investigate different kinds of scientifically and commercially available laser (yellow, red, and infrared) and LED stimulation systems for the first time using a chick embryo model under a fixed human cadaver (scalp and/or skull) and without this anatomical preparation.

\section{Materials and Methods}

\section{Chick Embryo Model}

Fertilized white leghorn chicken (Gallus domesticus L.) eggs (Schropper GmbH, Gloggnitz, Austria) were incubated for 3 days at $37.6^{\circ} \mathrm{C}$ and $70-75 \%$ relative humidity (J. Hemel Brutgeräte GmbH \& Co KG, Am Buschbach, Germany). On day 3, the eggs were opened into plastic weighing boats, covered with square Petri dishes and returned to the incubator. The experiments were performed on the chorioallantoic membrane (CAM) of 1-week-old chicken embryos. Only embryos that showed no bleeding or deformities were selected for the study. During the experiments, the weighing boats were placed on a heating pad at $37^{\circ} \mathrm{C}$ (Sunflower 68003; Soehnle, Murrhardt, Germany) and the lids were removed (fig. 1). 
Fig. 1. View of a 1-week-old shellless embryo showing the extraembryonic vessels (CAM).

Fig. 2. Laser experiments at the Institute of Pathophysiology and Immunology at the Medical University of Graz with laser stimulation equipment from the TCM Research Center Graz.
Litscher et al.: Transcranial Yellow, Red, and Infrared Laser and LED Stimulation: Changes of Vascular Parameters in a Chick Embryo Model
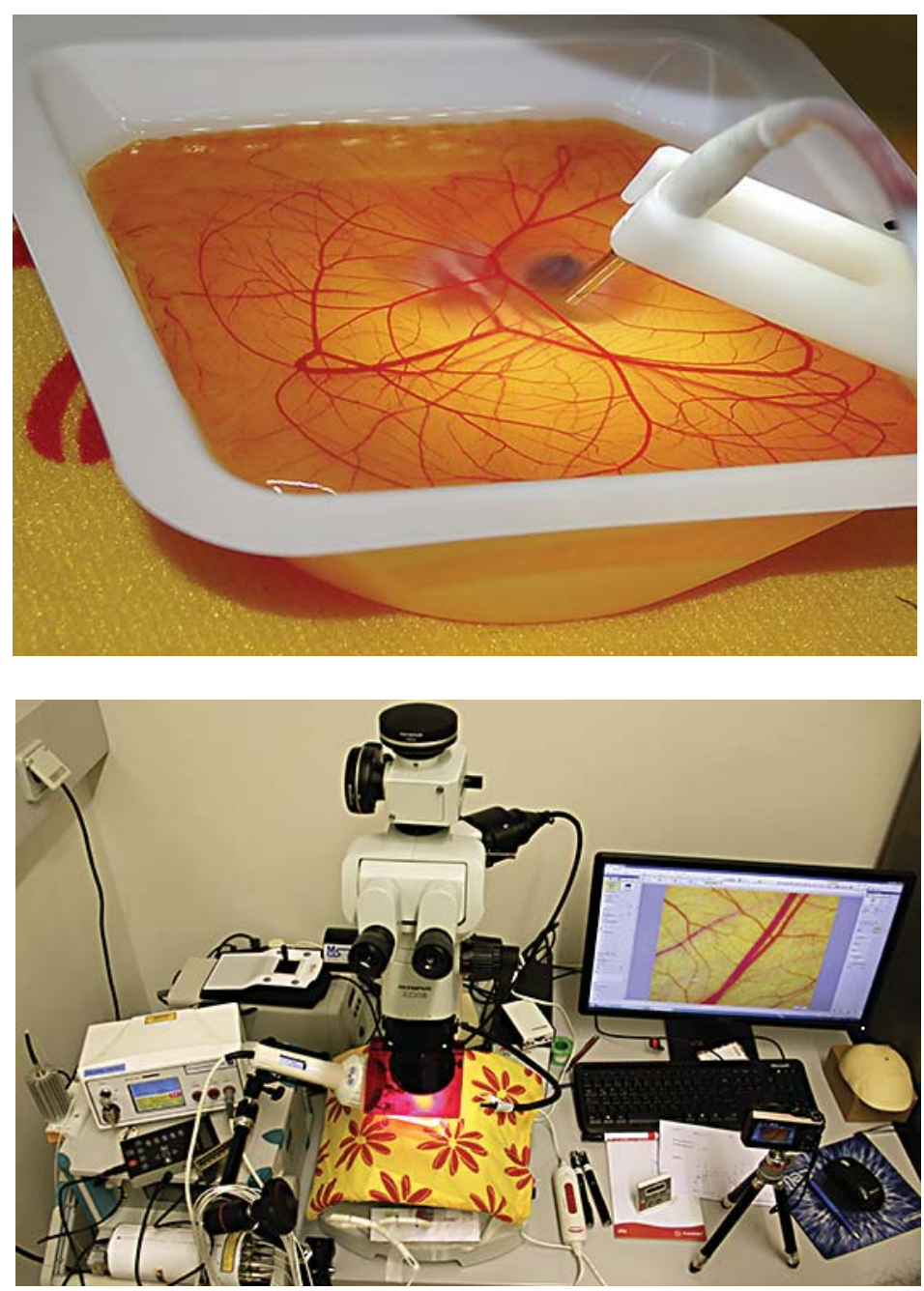

\section{Laser Stimulation}

For laser stimulation, three different systems were used.

Yellow laser stimulation was performed using a system currently available from Weber Medical (Endolaser, Lauenförde, Germany) and, for research purposes, at the Medical University of Graz. The wavelength of the yellow laser was $589 \mathrm{~nm}$, the output power $50 \mathrm{~mW}$, and the diameter of the laser needle was $500 \mu \mathrm{m}$. Stimulation duration was $10 \mathrm{~min}$ [15].

Multichannel red laser needle stimulation allows the noninvasive simultaneous stimulation of different points on the body. The laser needle method is based on systems with 8-12 separate semi-conductor laser diodes and emission wavelengths of $658 \mathrm{~nm}$. The system consists of flexible optical light fibers which conduct the laser light with minimal loss to the laser needle. Thus, a high optical density can be achieved at the distal end of the laser needle. The intensity of the laser needles is optimized in such a way that the volunteer or patient does not immediately feel the activation of the needle $(30-40 \mathrm{~mW}$ per needle; diameter $500 \mu \mathrm{m}$; duration 10 $\mathrm{min}$ ). More details regarding this method are described in previous publications [16].

In addition, a Physiolaser (Reimers \& Janssen, Waldkirch, Germany) with a wavelength of $904 \mathrm{~nm}$ and an output power of $90 \mathrm{~W}$ (multi-cluster superpulse; $5 \times 30 \mathrm{~W} ; 200 \mathrm{~ns} ; 2 \mathrm{~J} /$ $7.4 \mathrm{~s}$ at $9,000 \mathrm{~Hz}$ ) was used. The manufacturers propose to use this system also for transcranial stimulation. 
Litscher et al.: Transcranial Yellow, Red, and Infrared Laser and LED Stimulation:

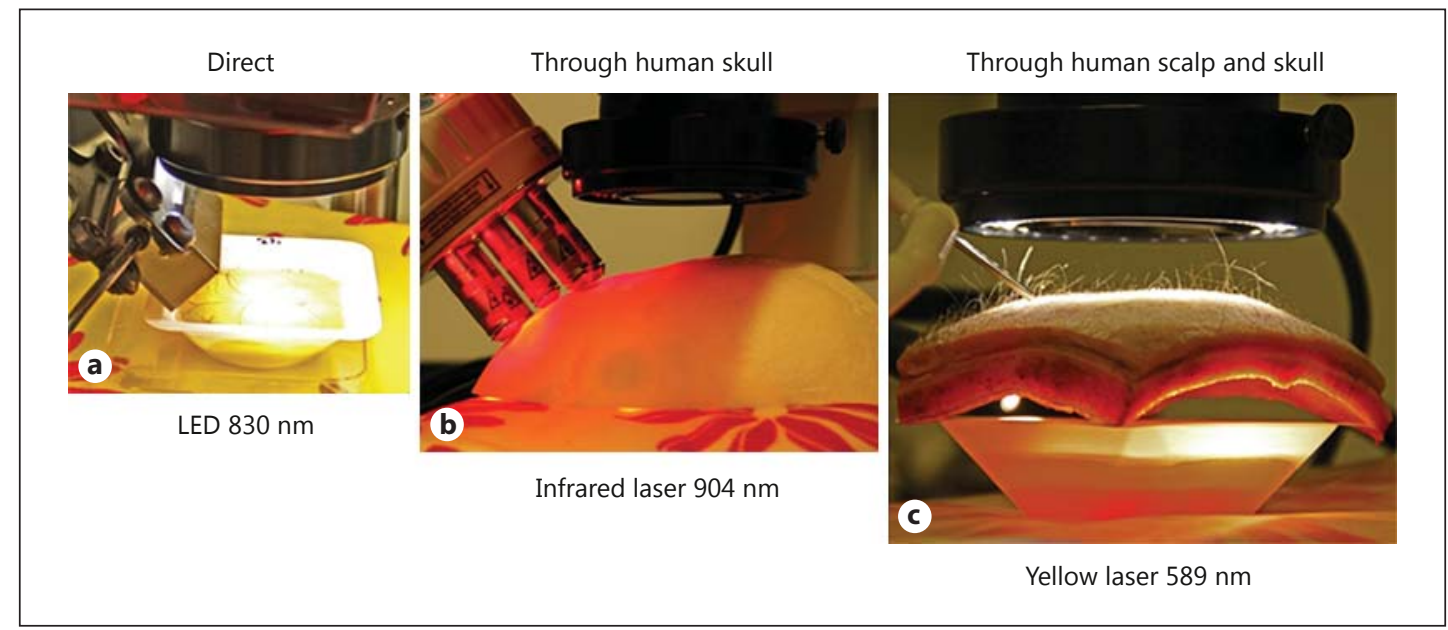

Fig. 3. LED (a) and laser stimulation (b, c) in a chick embryo model. a Direct LED radiation. b Multi-cluster superpulse infrared laser stimulation through a human skull (the CAM model is underneath the skull). c Yellow laser stimulation through a human scalp and skull.

Fig. 4. Experimental protocol for laser/LED stimulation and recording profile. Altogether, a recording session of $17 \mathrm{~min}$ was performed in each experiment.

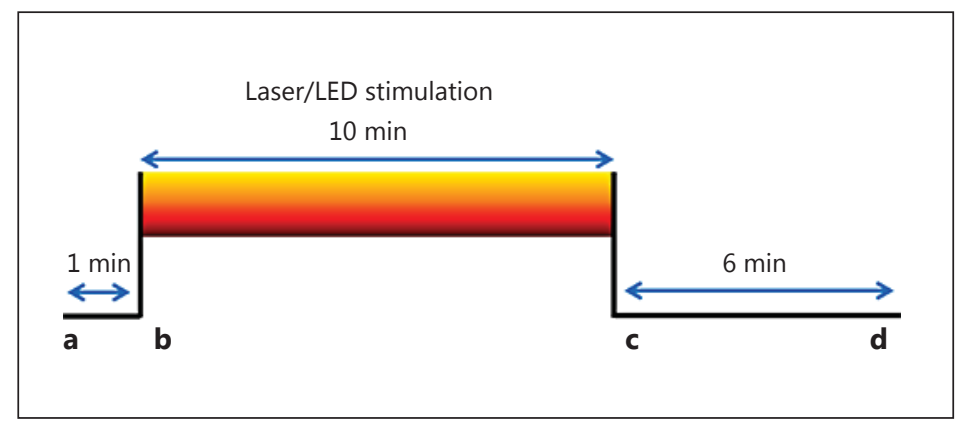

Last but not least, a new LED-based system called medlouxx (Laneg GmbH, Schönwalde, Germany) was investigated. This system emits noncoherent infrared LED radiation with a wavelength of $830 \mathrm{~nm}$. The output power of the LED applicator is $760 \mathrm{~mW}$, and the LED class of the system is $1 \mathrm{M}$ according to IEC60825-1:A2. The absolute value of the measurement inaccuracy concerning the LED output power is $20 \%$ [3].

All three systems as well as the measurement methods are shown in figure 2 . In addition, the different applications of the stimulation methods (direct, through the human skull, and through the human scalp and skull) are demonstrated in figure 3.

\section{Procedure}

The measurement profile and times are shown schematically in figure $4 \mathrm{a}-\mathrm{d}$. Four measurement periods were compared: before stimulation (fig. 4a), immediately after stimulation onset (fig. 4b), immediately after stimulation was turned off (fig. 4c), and 6 min after stimulation had ended (fig. 4d).

\section{Measurement Methods}

The measurements were carried out in November and December 2014 using an innovative microscope system that has been available at the Institute of Pathophysiology and 
Litscher et al.: Transcranial Yellow, Red, and Infrared Laser and LED Stimulation:

Fig. 5. Image sequences before and after direct LED stimulation (a) and LED stimulation through a human skull (b). Note the percent changes of the processed area of the vessel.

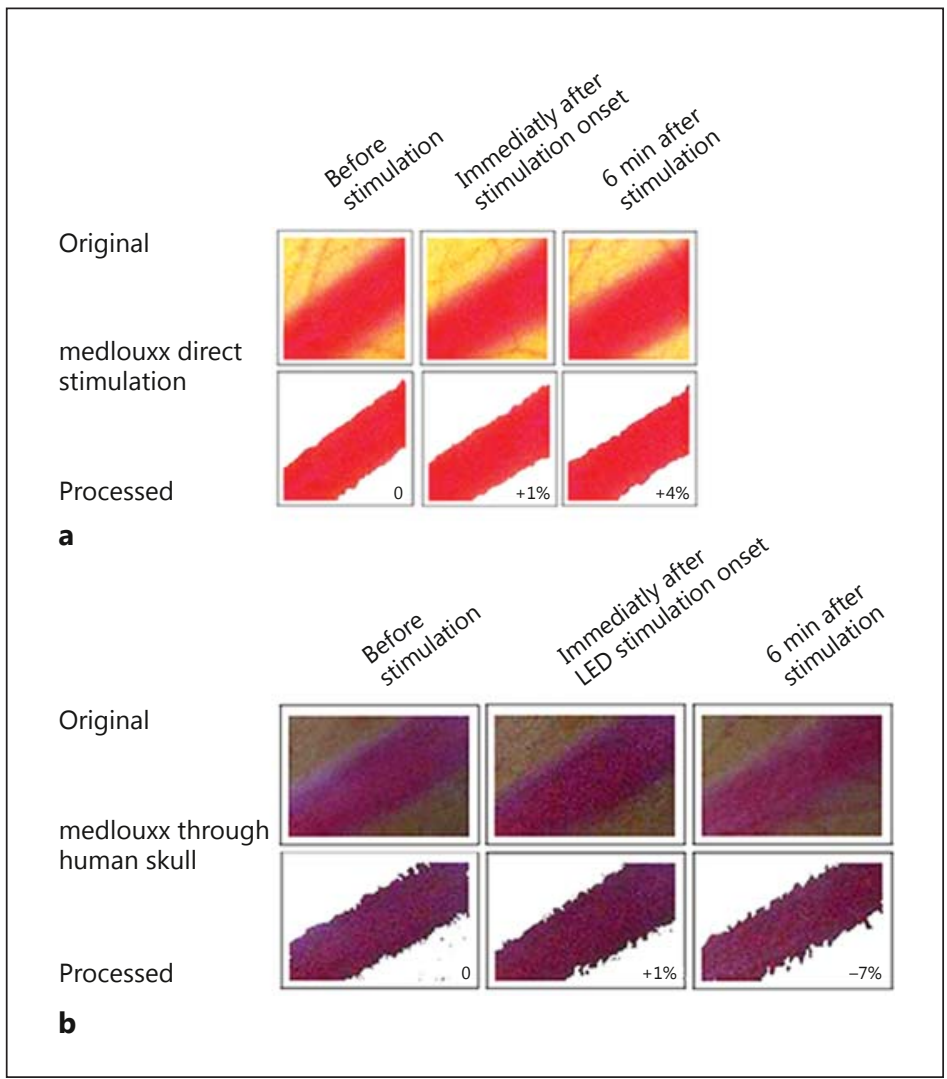

Immunology at the Medical University of Graz as of July 22, 2014: an Olympus coded fluorescence stereomicroscope SZX16 and the dimension software package (Olympus, Shinjuku, Tokyo, Japan; fig. 2).

With this research stereomicroscope, image acquisition of blood vessels including video acquisition of blood circulation is possible at a very high resolution (zoom range $\times 7.0-115$; numerical aperture 0.3).

\section{Mathematical Analysis}

Special image processing programs developed at the Beijing University of Science and Technology were used for data analysis $[17,18]$. The image processing procedure can be described as follows. Firstly, an interesting region including one small blood vessel is chosen. Secondly, we extract the vessel as shown in figures 5-7 and get the subfigure of the vessel. Thirdly, we use a matting algorithm to process the subfigures. Fourthly, we then calculate the area of the blood vessel by pixels. Fifthly and finally, we give statistic results for the area of the blood vessel. These results serve as estimated values of the blood volume within the vessel. Percent changes are given in the results.

\section{Results}

Figures 5-9 show image sequences to illustrate the changes of blood flow volume within the small vessels. In figure $5 \mathrm{a}$, the alterations after direct LED stimulation using the medlouxx system are demonstrated. The original photos are shown in the upper row, and the processed 
Integrative

Medicine

International

Fig. 6. Original and processed images after direct infrared Physiolaser stimulation (a) and after stimulation through a human skull (b). Note the increase in blood volume (area of the vessel) $1 \mathrm{~min}(+7 \%)$ and $6 \mathrm{~min}(+17 \%)$ after stimulation through the skull.

\begin{tabular}{l|l}
\hline Integr Med Int 2015;2:80-89 \\
\hline DOI: 10.1159/000431176 & $\begin{array}{l}\text { C 2015 S. Karger AG, Basel } \\
\text { www.karger.com/imi }\end{array}$ \\
\hline
\end{tabular}

Litscher et al.: Transcranial Yellow, Red, and Infrared Laser and LED Stimulation: Changes of Vascular Parameters in a Chick Embryo Model

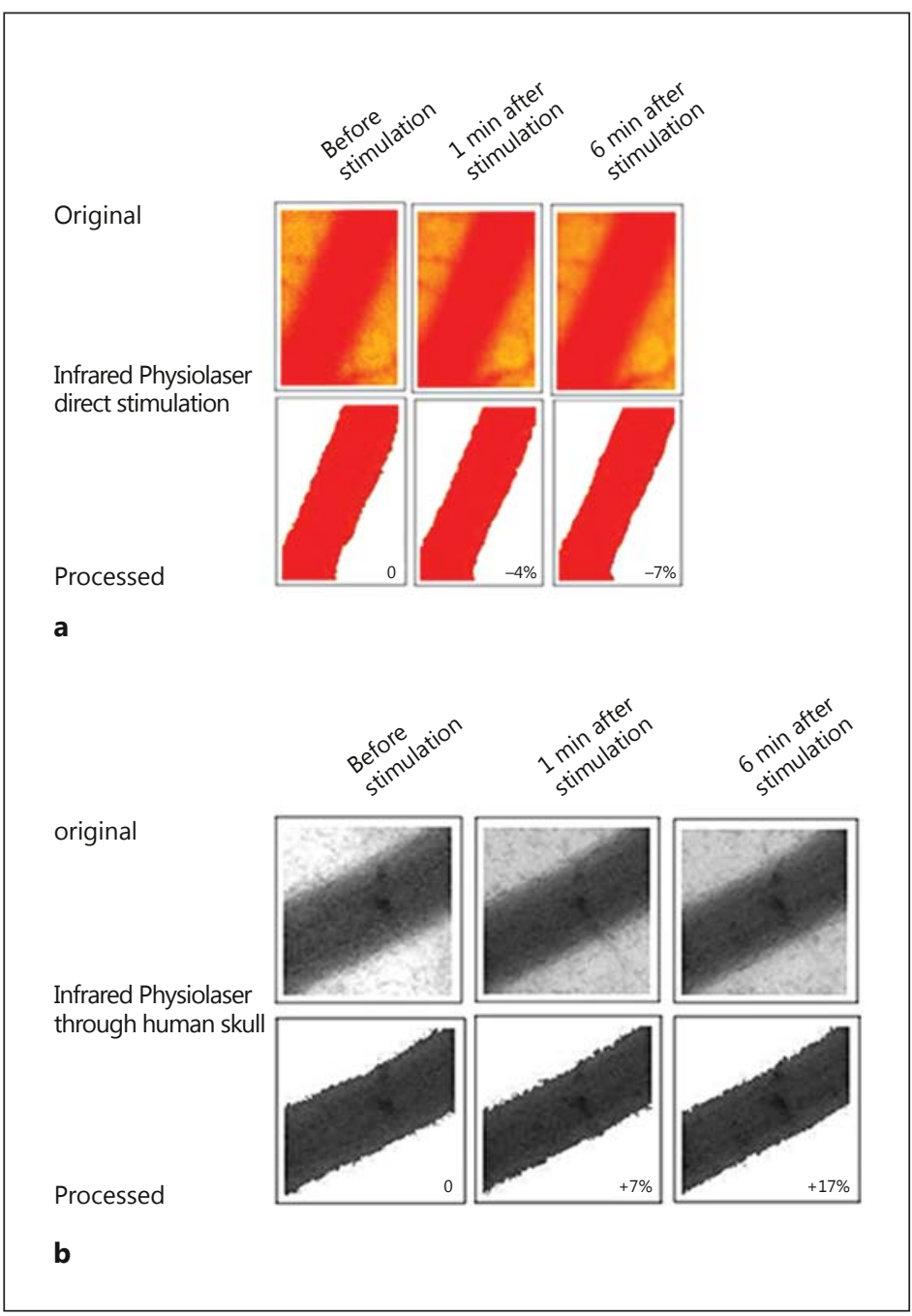

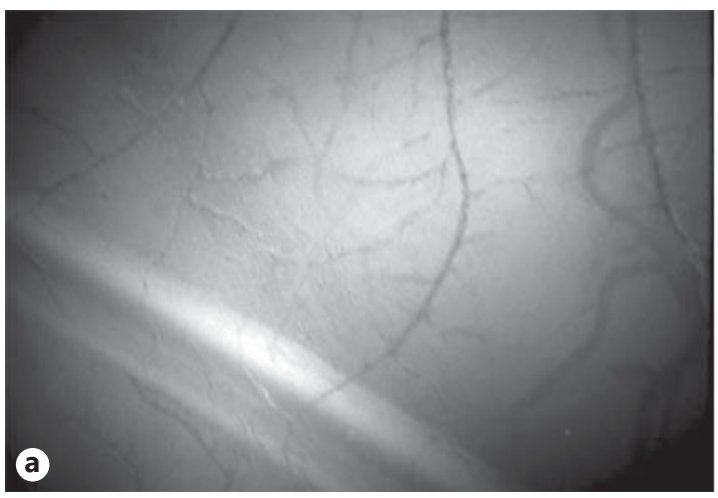

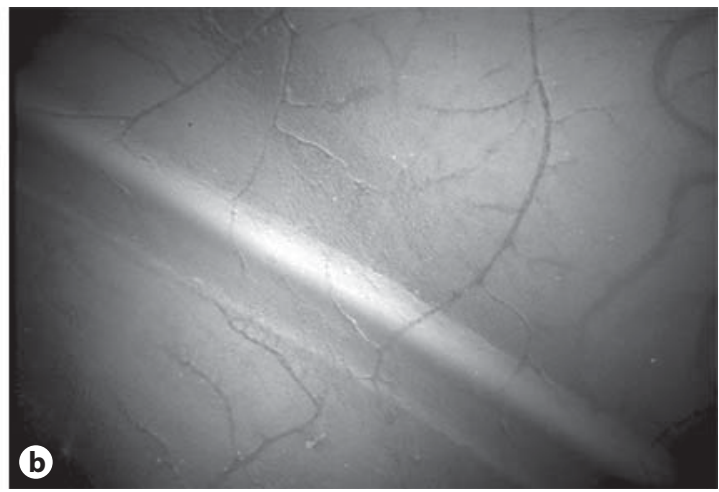

Fig. 7. Two pictures from 904-nm laser stimulation through the skull before (a) and immediately after stimulation (b). 

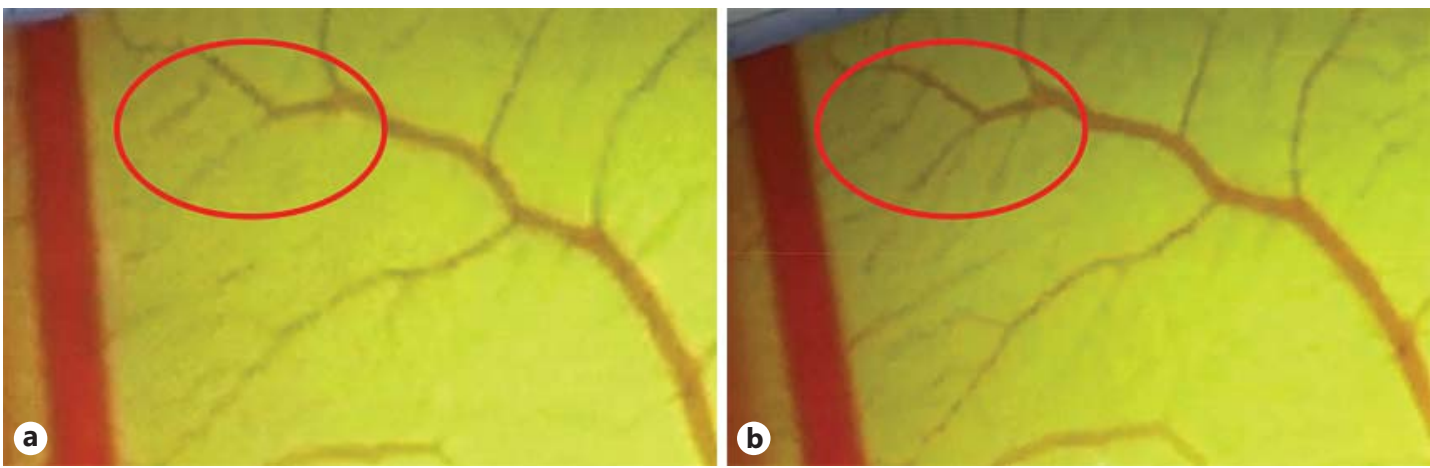

Fig. 8. Two pictures from 589-nm laser stimulation before (a) and 6 min after stimulation (b). Note the increase in the vessels (red ellipse).

Fig. 9. Yellow laser stimulation in a CAM model. Note the decrease in the vessel diameter after direct laser stimulation (a) and the increase after stimulation through a human skull (b).

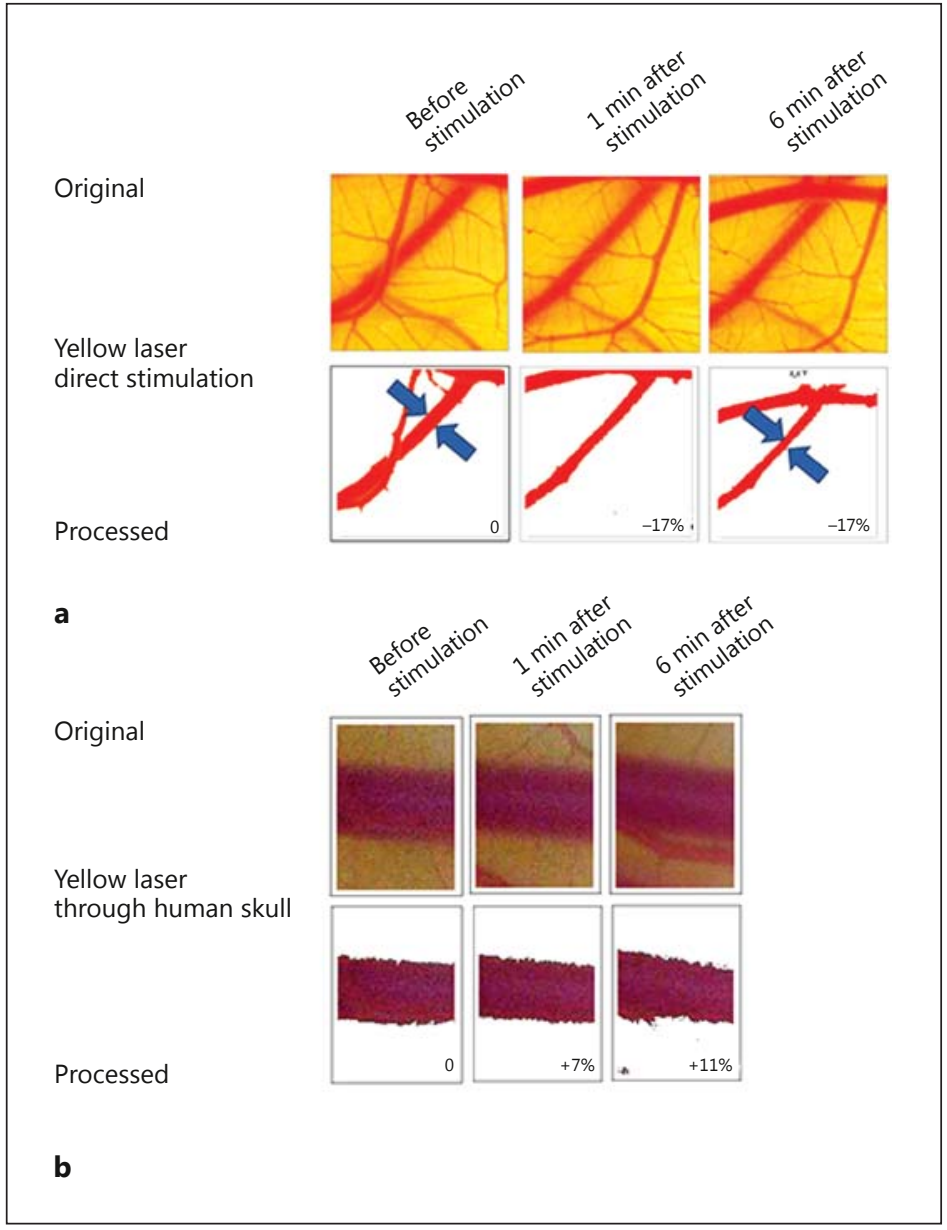

photos are directly below the original images. Figure $5 b$ shows the results of LED radiation through a human skull.

The images in figure 6 were taken before and after infrared laser stimulation (Physiolaser). Again, the pictures show measurements after direct stimulation (fig. 6a) and after stimulation through a human skull (fig. 6b). 
Litscher et al.: Transcranial Yellow, Red, and Infrared Laser and LED Stimulation:

Changes of Vascular Parameters in a Chick Embryo Model

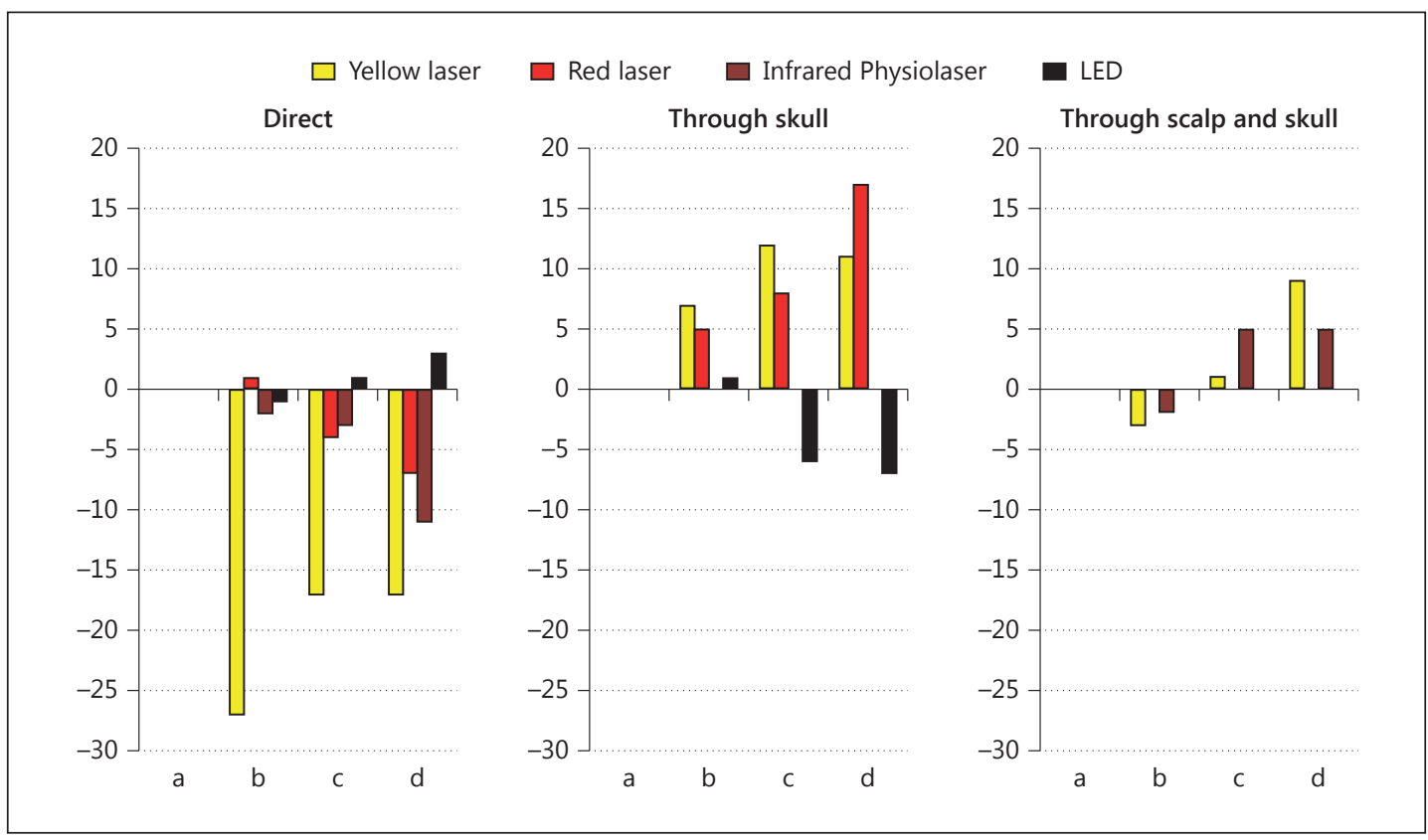

Fig. 10. Transcranial laser and LED stimulation (direct, through a human skull, and through a human scalp and skull) are shown with regard to the experimental protocol (a-d). Compare also figure 4.

Figures 7 and 8 illustrate the laser effects. Figure 7 demonstrates that it is very hard to quantify the changes in the vasculature without mathematical analysis of the photo. The photos before and after stimulation with the infrared laser (904 $\mathrm{nm}$ ) look very similar. However, when comparing the vessels to those shown in figure $6 \mathrm{~b}$, an increase of about $7 \%$ can be seen in the blood vessels (fig. 7). Figure 8 also shows the increase in blood vessels after yellow laser stimulation. The pictures were taken before and $6 \mathrm{~min}$ after laser stimulation.

A part of the vascular system of the CAM is also presented in figure 9a. After mathematical processing, the changes in the diameter of one of the vessels $(-17 \%)$ and, therefore, the initial vasoconstrictive effect after direct yellow laser stimulation can be clearly seen. On the other hand, yellow laser stimulation through a human skull does not induce similar effects. Moreover, an increase in blood volume could be observed after processing the images (fig. 9b).

Figure 10 summarizes the results obtained within this pilot study. In addition to the kind of stimulation (direct, through the skull, and through the scalp and skull), the percent changes of the vessels after mathematical processing of the images are shown.

\section{Discussion}

The CAM model is a preclinical model widely used for vascular and antivascular effects of therapeutic agents in vivo [19]. It is one of the most well-characterized and useful models. The density of the capillary network of the extra-embryonic CAM is easily accessible and can be used to investigate angiogenesis [20]. Fertilized chicken eggs are suggested as an alternative to mammalian models: the CAM is free of nerve cells; moreover, in our study we used 1-week-old embryos, which have not yet developed the ability to experience pain [21].

The aim of this work was to evaluate the influence of laser or LED radiation in the range of yellow, red, or infrared light on the changes of blood vessels. Therefore direct radiation, radiation through a human skull, and radiation through a human scalp and skull was performed. 
Direct yellow laser stimulation resulted in vasoconstriction, which was extremely pronounced immediately after yellow laser stimulation onset (fig. 10). The effect was still present after 10 min of yellow laser stimulation, and also 6 min after the laser had been turned off, although it had worn off a bit. A different effect could be seen following direct stimulation of the CAM model using red laser $(658 \mathrm{~nm})$ or infrared laser $(904 \mathrm{~nm})$ with different output powers, and also during and after LED stimulation (fig. 10). It seems that the yellow laser has an extremely vascular effect, which at this moment is not easy to explain in detail (these are the first data on this topic). However, previous investigations in human adults have recently shown that yellow laser acupuncture stimulation can have an influence on blood pressure and also on other related biological parameters like heart rate or heart rate variability [15].

The CAM model has been used for basic research in different applications. However, it has only been used in very few studies in combination with laser therapy. One example is to modulate the rapid growth of blood vessels in the wet form of age-related macular degeneration [22]. The results of our present study show clearly that there are changes in the blood vessels, not only caused by direct stimulation, but also during and after stimulation through a human skull and even through a human scalp and skull. These results are very important for a possible transcranial laser therapy. Stroke, dementia, Alzheimer's disease, and Parkinson's disease are only a few important indications for transcranial laser therapy. It is interesting that direct yellow laser stimulation of the blood vessels resulted in initial vasoconstriction, whereas stimulation through the scalp and skull mostly induced vasodilation. This was not the case when LED stimulation was used (fig. 10, stimulation through the skull).

It has to be mentioned that near-infrared light can also modulate numerous cellular functions. For example, positive effects include acceleration of wound healing, improved recovery from ischemic injury, and attenuated degeneration of injured optic nerves by improving mitochondrial energy metabolism and production [23].

The results of this study are also important because they are in close relationship with previous investigations at the Medical University of Graz. These studies deal with high-tech acupuncture, cerebral circulation, and microcirculation in humans [24, 25]. Transcranial Doppler sonography has been extensively used in various basic and clinical situations, and over the last decade it has also established its role as a tool for investigating the effects of different acupuncture methods [24].

Already in 1997, our team was able to scientifically prove that acupuncture needles can increase blood flow velocity in the brain $[26,27]$. The computer- and robot-controlled biosensors and probes integrated in a special helmet construction, coupled with light, ultrasound, and highly sensitive bioelectrical monitoring methods yielded reproducible results and, for the first time, confirmed specific effects of acupuncture in the brain [27]. Now, with the help of the CAM model, we can also verify effects on blood vessels in a model and we can separate the influence of the different layers (skin, scalp, and skull) during laser stimulation in the head. This will bring us forward step by step in the investigation of transcranial laser stimulation effects, and it will also open up new therapies for lifestyle-related diseases such as stroke, dementia, Alzheimer's and maybe Parkinson's disease.

\section{Acknowledgements}

The study was supported by the German Academy of Acupuncture, the Science Department of the City of Graz, the Austrian Federal Ministries of Science, Research, Economy, and Health, and Eurasia-Pacific Uninet who provided the 3-month scholarship in Austria for Prof. Guangjun Wang. We gratefully thank SFL Technologies (Stallhofen, Austria) for providing the Olympus SZX16 stereomicroscope. 
Litscher et al.: Transcranial Yellow, Red, and Infrared Laser and LED Stimulation:

Changes of Vascular Parameters in a Chick Embryo Model

\section{Disclosure Statement}

The authors declare that there is no conflict of interest regarding the publication of this paper.

\section{References}

1 Pan WD, Zhou H: Inclusion of integrative medicine in clinical practice. Integr Med Int 2014;1:1-4.

-2 Litscher G: Innovative integrative medicine. Integr Med Int 2014;1:65-66.

-3 Litscher D, Litscher G: Laser therapy and dementia: a database analysis and future aspects on LED-based systems. Int J Photoenergy 2014;2014:268354.

$>4$ Pan WD: Integrative reserve and integrative medicine. Integr Med Int 2014;1:127-129.

5 Rojas JC, Gonzalez-Lima F: Neurological and psychological applications of transcranial lasers and LEDs. Biochem Pharmacol 2013;86:447-457.

-6 Xuan W, Vatansever F, Huang L, Hamblin MR: Transcranial low-level laser therapy enhances learning, memory, and neuroprogenitor cells after traumatic brain injury in mice. J Biomed Opt 2014;19:108003.

7 Hacke W, Schellinger PD, Albers GW, Bornstein NM, Dahlof BL, Fulton R, Kasner SE, Shuaib A, Richieri SP, Dilly SG, Zivin J, Lees KR; NEST 3 Committees and Investigators: Transcranial laser therapy in acute stroke treatment: results of neurothera effectiveness and safety trial 3, a phase III clinical end point device trial. Stroke 2014;45:3187-3193.

8 Litscher D, Litscher G: Laser therapy and stroke: quantification of methodological requirements in consideration of yellow laser. Int J Photoenergy 2013;2013:575798.

-9 Wan S, Parrish JA, Anderson RR, Madden M: Transmittance of nonionizing radiation in human tissues. Photochem Photobiol 1981;34:679-681.

$\checkmark 10$ Tedford CE, DeLapp S, Jacques S, Anders J: Quantitative analysis of transcranial and intraparenchymal light penetration in human cadaver brain tissue. Lasers Surg Med 2015;47:312-322.

11 Bevilacqua F, Piguet D, Marquet P, Gross JD, Tromberg BJ, Depeursinge C: In vivo local determination of tissue optical properties: applications to human brain. Appl Opt 1999;38:4939-4950.

-12 Bashkatov AN, Genina EA, Kochubey VI, Tuchin VV: Optical properties of human cranial bone in the spectral range from 800 to $2000 \mathrm{~nm}$. Proc SPIE 6163, Saratov Fall Meeting 2005: Optical Technologies in Biophysics and Medicine VII, 616310 (July 21, 2006).

13 Stolik S, Delgado JA, Pérez A, Anasagasti L: Measurement of the penetration depths of red and infrared light in human 'ex vivo' tissues. J Photochem Photobiol B 2000;57:90-93.

14 Jagdeo JR, Adams LE, Brody NI, Siegel DM: Transcranial red and near infrared light transmission in a cadaveric model. PLoS One 2012;7:e47460.

15 Litscher D, Wang GJ, Gaischek I, Wang L, Wallner-Liebmann S, Petek E: Yellow laser acupuncture - a new option for prevention and early intervention of lifestyle-related diseases: a randomized, placebo-controlled trial in volunteers. Laser Ther 2015;24:53-61.

16 Litscher G: Integrative laser medicine and high-tech acupuncture at the Medical University of Graz, Austria, Europe. Evid Based Complement Alternat Med 2012;2012:103109.

17 Zhang M, Min LQ, Zhang Q, Liu S: Matting algorithm and application based on Mahalanobis distance and the fuzzy C-means clustering algorithm. J Univ Sci Technol B 2014;36:688-694.

18 Li M, Min LQ, Litscher G: An automatical algorithm based on local image properties. J Math Imaging Vis. Submitted.

19 Kue CS, Tan KY, Lam ML, Lee HB: Chick embryo chorioallantoic membrane (CAM): an alternative predictive model in acute toxicological studies for anti-cancer drugs. Exp Anim 2015;64:129-138.

20 Ribatti D: Chick embryo chorioallantoic membrane as a useful tool to study angiogenesis. Int Rev Cell Mol Biol 2008;270:181-224.

21 Aleksandrowicz E, Herr I: Ethical euthanasia and short-term anesthesia of the chick embryo. ALTEX 2015;32: 143-147.

22 Samkoe KS, Cramb DT: Application of an ex ovo chicken chorioallantoic membrane model for two-photon excitation photodynamic therapy of age-related macular degeneration. J Biomed Opt 2003;8:410-417.

-23 DeSmet KD, Paz DA, Corry JJ, Eells JT, Wong-Riley MTT, Henry MM, Buchmann EV, Connelly MP, Dovi JV, Liang HL, Henshel DS, Yeager RL, Millsap DS, Lim J, Gould LJ, Das R, Jett M, Hodgson BD, Margolis D, Whelan HT: Clinical and experimental applications of NIR-LED photobiomodulation. Photomed Laser Surg 2006;24:121128.

-24 Litscher G: High-tech acupuncture made in Austria - cerebral circulation. J Chin Int Med 2012;10:362-368.

25 Litscher G: Bioengineering assessment of acupuncture, part 2: monitoring of microcirculation. Crit Rev Biomed Eng 2006;34:273-293.

26 Litscher G, Schwarz G, Sandner-Kiesling A, Hadolt I, Eger E: Effects of acupuncture on the oxygenation of cerebral tissue. Neurol Res 1998;20(suppl 1):S28-S32.

27 Litscher G, Schwarz G, Sandner-Kiesling A, Hadolt I: Robotic transcranial Doppler sonography probes and acupuncture. Int J Neurosci 1998;95:1-15. 